\title{
Capões como Ilhas para Artrópodes no Pantanal
}

\author{
Rodrigo Aranda
}

Universidade Federal de Mato Grosso do Sul, e-mail: rodrigoaranda.biologo@gmail.com

\section{EntomoBrasilis 6 (3): 173-177 (2013)}

\begin{abstract}
Resumo. A configuração do habitat é um dos fatores que pode influenciar a distribuição dos organismos. O pantanal por apresentar mosaico natural de formações se enquadra para testar tais afirmações. O presente estudo teve como objetivo avaliar se a distância e o tamanho de capões afetam a composição da comunidade de artrópodes testando a hipótese de que quanto maior e mais próximo o capão da mata ciliar, maior sua diversidade. Foram amostradas seis parcelas de $30 \mathrm{~cm}^{2}$ em nove capões onde a serrapilheira e o solo foram coletados, acondicionados em sacos plásticos e posteriormente triados. Houve relação entre a composição da comunidade de artrópodes em relação à distância e o tamanho indicando que o pulso de inundação do último ano afetou de forma significativa a comunidade de artrópodes de solo em capões. O acompanhamento dos processos de recolonização dos capões é sugerido.
\end{abstract}

Palavras-Chave: Artrópodes de solo; Fragmentação; Metacomunidade; Serrapilheira.

\section{Capons as Island for Arthropods in Pantanal}

Abstract. The configuration of habitat is one of the factors that influence the distribution of organisms. The Pantanal by presenting mosaic natural formations fit to test such claims. The present study aimed to evaluate the distance and size of riparian capons affect the community composition of arthropods tested the hypothesis that the higher and closer to the capon of riparian vegetation, the greater its diversity. We sampled six plots of $30 \mathrm{~cm}^{2}$ in nine capons where the leaf-litter and soil were collected, placed in plastic bags and later sorted. There was a relationship between the composition of arthropod community in relation to distance and size indicating that the flood pulse of the last year significantly affected the community of soil arthropods in geldings. The monitoring of the processes of recolonization of the capons is suggested.

Key-words: Fragmentation; Leaf-litter; Metacommunity; Soil arthropods.

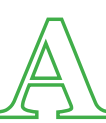
relação entre espécie e área é bem conhecida em muitos grupos e as teorias de biogeografia de ilhas (MACARTHUR \& WILSON 1967) e metapopulações (Levins 1969) podem ser aplicadas em ambientes continentais fragmentados com a mesma valia. A configuração do habitat (isolamento, distância, tamanho, arranjo espacial e nível de fragmentação) tem sido enfatizada para várias espécies, em escalas de tempo e de espaço, sendo a distribuição dos organismos influenciada diretamente pela configuração do habitat (KRAWCHUK \& TAYLOR 2003). Artrópodes em regiões de ilhas parecem se enquadrar neste modelo (STRONG JR. \& ReY 1982), mas em outros ambientes fragmentados essas relações são pouco estudadas (DEBINSKI \& Holt 2000).

Os efeitos da estrutura do habitat e fragmentação atuam de forma diferente em grupos funcionais distintos de insetos (HunTer 2002) e outros artrópodes (Iluig et al. 2010), porém, ainda há pouca informação sobre a dinâmica das comunidades de solo, fazendo-se necessário maior esforço para compreensão dos processos que definem as comunidades (CORREIA 2002). A conservação de grupos, como os insetos, vem sendo uma das prioridades por ser um grupo com a maioria das espécies ainda não descritas e por serem bem representativo nos ecossistemas terrestre e aquático (HuGHES et al. 2000) e demonstrar de forma razoável o efeito do impacto e da fragmentação, devido as diversas funções desempenhadas no ecossistema (McInTYre et al. 2001).

O pantanal é um mosaico de ambientes, onde o pulso de inundação é a principal força estruturadora das comunidades (Junk et al. 1989). As enchentes anuais alagam uma vasta área da planície durante meses. Um pequeno gradiente altitudinal de poucos metros permite que áreas mais elevadas não sejam alagadas, permanecendo como ilhas na planície inundada. Popularmente conhecidos como "capões", esses fragmentos naturais se enquadram plenamente como modelo para testar relações de espécie-área de forma natural (Bordignon et al. 2007) e compreender como os padrões de distribuição das espécies é afetado em ambientes fragmentados e com efeitos de distúrbios sazonais bem definido. Mesmo sendo um ambiente propício para esses estudos, o Pantanal ainda é pouco estudado em relação ao comportamento da fauna edáfica (CORRÊA et al. 2006; BATTIROLA et al. 2010) sendo dado mais enfoque a fauna associada à árvores ou dossel (Battirola et al. 2004, 2007; Саstilho et al. 2005; Marques et al. 2006).

Este trabalho teve como objetivo avaliar o efeito do tamanho dos capões e a distância destes da mata ciliar sobre a comunidade de artrópodes de solo no Pantanal, testando a hipótese de que capões maiores e mais próximos da mata ciliar apresentaram maior riqueza e abundância, assim como maior similaridade na composição de artrópodes de solo.

\section{MATERIAL E MÉTODOS}

O estudo foi realizado no Pantanal na região do MirandaAbobral, próximo a Base de Estudos do Pantanal (19 $34^{\circ}$ '34,84" S e $57^{\circ} 1^{\prime} 6,98^{\prime \prime}$ ), da Universidade Federal de Mato Grosso do Sul, Corumbá, MS em outubro de 2011. Foram amostrados nove capões, e em cada um, foram coletadas seis parcelas de $30 \mathrm{~cm}^{2}$ de serrapilheira e parte do solo, distribuída ao acaso. O conjunto das seis parcelas foi considerado uma unidade amostral para cada capão. As amostras de serrapilheira e parte do solo de cada parcela foram acondicionadas em sacos plásticos e armazenadas 
sob refrigeração para preservação do material até triagem. No laboratório, o material foi peneirado (malha 1,5 mm). Em uma bandeja plástica de $50 \times 30 \times 8 \mathrm{~cm}$, sendo os artrópodes triados, acondicionados em frascos contendo álcool $70 \%$ para posterior identificação, através da utilização de chaves dicotômicas especificas (Borror \& DeLong 1988) até o nível de família para os insetos e Classe ou Ordem para demais grupos de artrópodes. Os dados de tamanho e distância dos capões em relação à mata ciliar foram obtidos com auxílio de imagens de satélites através de vetorização para cálculo da área (hectare) e distância linear $(\mathrm{km})$ da mata ciliar (Zonummaps for Google Earth).

Foi calculado para cada capão o índice de diversidade de Shanonn-Wiener (H'), regressão linear entre a abundância e a área, regressão cúbica entre a abundância de artrópodes contra a distância da mata ciliar e a riqueza de morfoespécies e área e regressão quadrática entre riqueza de morfoespécies contra distância até a mata ciliar. Foi realizado escalonamento multidimensional não métrico (NMDS) (similaridade de BrayCurtis) para verificar se o tamanho dos capões e a distância da mata ciliar influenciam a composição da comunidade de artrópodes de solo.

\section{RESULTADOS E DISCUSSÃO}

Coletou-se 290 indivíduos distribuídos em 69 morfoespécies (Tabela 1). O menor capão apresentou área de 0,40 ha e o maior 1,54 há (média $0,73 \pm \mathrm{DP}$ 0,14) e a distância da mata ciliar variou de $0,51 \mathrm{~km}$ a $1,83 \mathrm{~km}$ (média $1,18 \pm$ DP 0,16 ) (Tabela 2). O capão que apresentou maior diversidade foi o capão $5(\mathrm{H}=1,27)$ que possui uma das maiores áreas e menores distâncias da mata ciliar e o que apresentou menor diversidade foi o capão $4(\mathrm{H}=0,76)$, com uma das menores áreas, maior distância da mata ciliar e ainda intensa perturbação por pisoteio de gado.

Avaliando a área dos capões e a distância da mata ciliar como preditores de abundância e riqueza, tivemos uma relação significativa entre as variáveis isoladamente (Figura 1A-1D). Quando avaliamos o NMDS da composição das morfoespécies, podemos observar o agrupamento de capões maiores e mais próximos e de menores e mais distantes (Figura 2A). Este padrão se torna mais claro quando se realiza o NMDS excluindo o capão 4 das análises (Figura 2B) por ser um capão altamente perturbado por pisoteio de gado.

A diminuição relativa da abundância dos indivíduos em relação ao tamanho (Figura 1A) pode estar relacionada com o esforço amostral não equitativo em relação ao tamanho, uma vez que todos os capões tiveram o mesmo número de amostras. O aumento da abundância em relação à distância (Figura1B) está relacionado com o predomínio de Coleoptera nas amostras.

Coleoptera foi o grupo mais abundante (60\%), representado principalmente por larvas de Tenebrionidae (48,27\%), assim como adultos de Chrysomelidae (2,75\%) que são decompositores de matéria orgânica.

As aranhas constituíram o segundo grupo mais representado (10,68\%), principalmente nos capões maiores e mais próximos (capões 5,8 e 9). Por serem um grupo trófico mais elevado, predadores, muitas vezes especializados, assim como Pseudoescorpiões $(4,82 \%)$ e Chilopoda $(1,37 \%)$ indicando que estes capões apresentam uma composição da comunidade mais estável para manter predadores. Grupos com as aranhas podem se deslocar verticalmente para áreas mais altas e a comunidade sustentar composições diferentes, contribuindo para a diversidade (BATTIROLA et al. 2010).

Os grupos com maior poder de dispersão e ampla representatividade funcional, como Coleoptera e Hymenoptera, foram capazes de chegar a capões mais distantes, enquanto grupos com baixa capacidade de dispersão, mas importantes decompositores, porém, com abundâncias representativas em fauna de solo (Gomes et al. 2007; Illig et al. 2010), como Collembola, Acari e Psocoptera, foram poucos frequentes (0,68\%; $1,72 \%$ e $6,55 \%$, respectivamente).

Isso pode estar relacionado com o pulso de inundação do ano de 2010 para 2011 sendo o maior pulso de inundação dos últimos 16 anos com 5,33 m segundo a régua fluviométrica de Ladário, MS que possivelmente homogeneizou a comunidade, onde os processos de recolonização ainda ocorriam. Esse fator pode ser verificado com a presença de Proctotrupidae (Hymenoptera), pequena vespa $(5-8 \mathrm{~mm})$ parasitóide de Coleoptera, altamente especializada e com baixa capacidade de dispersão, que foi amostrada apenas no capão seis que é o mais próximo da mata ciliar.

A composição dos grupos funcionais está diretamente relacionada com a formação da serrapilheira, quantidade, qualidade e umidade, sendo a fauna de solo um bom indicador da qualidade do solo e da estabilidade do ecossistema (Rubbo \& Kiesecker 2004; Souza et al. 2008) assim como características da estrutura da paisagem (KRAWCHUK \& TAYLOR 2003; CORRÊA et al. 2006). RiBAs et al. (2005) encontraram respostas diferentes em relação a composição da fauna edáfica em fragmentos de tamanhos diferentes, sendo favorecidos os grupos com maior capacidade de dispersão, enquanto grupos com menor capacidade de dispersão necessitam de áreas maiores e mais conectadas.

Poucos estudos com enfoque de como as comunidades se distribuem em relação a fatores biogeográficos foram realizados no Pantanal, tendo ênfase nas comunidades vegetais (Bordignon et al. 2007; Frizon 2007; Ротт \& РотT 2009). Para formações vegetais em capões não apenas o tamanho, a forma e o isolamento são fatores que afetam sua estrutura, mas também a matriz e histórico de perturbação das áreas vizinhas parecem ser fatores importantes (FrIZON 2007).

Desta forma, a comunidade de artrópodes de solo e serrapilheira respondem ao tamanho e a distância dos capões em relação a sua composição de espécies, grupos funcionais e ao processo de recolonização após uma perturbação sazonal mais marcante (pulso de inundação). O conhecimento do perfil da fauna de solo de capões no Pantanal se mostra uma área de grande interesse para compreensão de processos de dispersão e recolonização em ambientes naturais, devendo levar-se em consideração outras características como conectividade, qualidade das manchas, efeito da matriz, tempo para colonização, entre outras variáveis como moduladoras das comunidades de artrópodes de solo no Pantanal.

\section{AGRADECIMENTOS}

Ao Programa de Pós Graduação em Ecologia e Conservação da Universidade Federal de Mato Grosso do Sul e aos organizadores do Curso de Campo Ecologia do Pantanal edição 2011 pelo apoio logístico.

\section{REFERÊNCIAS}

Battirola, L.D., M.I. Marques, J. Adis \& A.D. Brescovit, 2004. Aspectos ecológicos da comunidade de Araneae (Arthropoda, Arachnida) em copas de palmeira Attalea phalerata Mart. (Arecaceae), no Pantanal de Poconé, Mato Grosso, Brasil. Revista Brasileira de Entomologia, 48: 421-430.

Battirola, L.D., J. Aids, M.I. Marques \& F.H.O. Silva, 2007. Comunidade de artrópodes associada à copa de Attalea phalerata Mart. (Arecaceae) durante o período de cheia no Pantanal de Poconé, MT. Neotropical Entomology, 36: 640651.

Battirola, L.D., M.I, Marques, A.D. Brescovit, G.H. RosadoNeto \& K.C. Anjos, 2010. Community of ground Araneae (Arthropoda, Arachnida) in a seasonally flooded forest in the Northern region of Pantanal of Mato Grosso, Brazil. Biota Neotropica. 10): disponível em: http://www. 
Tabela 1. Lista de morfoespécies amostradas em nove capões próximos a Base de Estudos do Pantanal ( $19^{\circ} 34^{\prime} 34,84^{\prime \prime}$ S e $57^{\circ} 1^{\prime} 6,98^{\prime \prime}$ O) Corumbá, MS, em outubro de 2011.

\begin{tabular}{|c|c|c|c|}
\hline Classe & Ordem & Morfoespécie & Capão \\
\hline \multirow[t]{20}{*}{ Arachnida } & Acarina & Acari sp1 & 2 \\
\hline & & Acari sp2 & $5,8,9$ \\
\hline & Araneida & Aranae sp1 & 1 \\
\hline & & Aranae sp2 & $1,2,3$ \\
\hline & & Aranae sp3 & 1,8 \\
\hline & & Aranae sp4 & 2,5 \\
\hline & & Aranae sp5 & $2,3,5$ \\
\hline & & Aranae sp6 & 3,8 \\
\hline & & Aranae sp7 & $3,8,9$ \\
\hline & & Aranae sp8 & 5 \\
\hline & & Aranae sp9 & 5 \\
\hline & & Aranae sp1o & 5 \\
\hline & & Aranae sp11 & 5 \\
\hline & & Aranae sp12 & 7,9 \\
\hline & Pseudoescorpionida & Pseudoescorpionidae sp1 & 5 \\
\hline & & Pseudoescorpionidae sp2 & 5 \\
\hline & & Pseudoescorpionidae sp3 & 5 \\
\hline & & Pseudoescorpionidae sp4 & $5,6,8,9$ \\
\hline & & Pseudoescorpionidae sp5 & 6,7 \\
\hline & & Pseudoescorpionidae sp6 & 6,9 \\
\hline \multirow[t]{2}{*}{ Chilopoda } & Geophilomorpha & Chilopoda sp1 & 1,6 \\
\hline & & Chilopoda sp2 & 6,7 \\
\hline Crustacea & Isopoda & Isopoda sp1 & 6 \\
\hline \multirow[t]{11}{*}{ Insecta } & Blatodea & Blatitdae sp1 & 6,9 \\
\hline & Collembola & Entomobryidae sp1 & 6,7 \\
\hline & Coleoptera & Carabidae sp1 & $1,3,7$ \\
\hline & & Coleoptera sp1 & 7 \\
\hline & & Coleoptera sp2 & 7,8 \\
\hline & & Coleoptera sp3 & 7 \\
\hline & & Chrysomelidae sp1 & 3 \\
\hline & & Chrysomelidae sp2 & 3 \\
\hline & & Chrysomelidae sp3 & 3 \\
\hline & & Chrysomelidae sp4 & 4,8 \\
\hline & & Chrysomelidae sp5 & 5 \\
\hline
\end{tabular}

\begin{tabular}{|c|c|c|c|}
\hline Classe & Ordem & Morfoespécie & Capão \\
\hline & & Chrysomelidae sp6 & 7 \\
\hline & & Curcullionidae sp1 & 5 \\
\hline & & Dermestidae sp1 & $2,5,6$ \\
\hline & & Dermestidae sp2 & 3,8 \\
\hline & & Nitidulidae sp1 & 2 \\
\hline & & Scarabaeidae sp1 & 5 \\
\hline & & Staphylinidae sp1 & 1 \\
\hline & & Staphylinidae sp2 & 3 \\
\hline & & Staphylinidae sp3 & $3,8,9$ \\
\hline & & Staphylinidae sp4 & 4 \\
\hline & & Staphylinidae sp5 & $4,5,6$ \\
\hline & & Tenebrionidae sp1 & $\begin{array}{l}1,2,3,5,6, \\
7,8,9\end{array}$ \\
\hline & & Tenebrionidae sp2 & $\begin{array}{l}1,2,3,5,6, \\
7,8\end{array}$ \\
\hline & & Tenebrionidae sp3 & $\begin{array}{c}2,3,5,6,7 \\
8,9\end{array}$ \\
\hline & & Tenebrionidae sp4 & $3,4,7$ \\
\hline & & Tenebrionidae sp5 & 3 \\
\hline & & Tenebrionidae sp6 & 5 \\
\hline & & Tenebrionidae sp7 & 5,6 \\
\hline & Diptera & Diptera sp1 & 1 \\
\hline & & Diptera sp2 & 2 \\
\hline & & Diptera sp3 & 2 \\
\hline & Hemiptera & Cicadellidae sp1 & $1,5,8$ \\
\hline & & Tingidae sp1 & 1 \\
\hline & Hymenoptera & Formicidae sp1 & $3,6,7,8,9$ \\
\hline & & Formicidae sp2 & $4,5,6$ \\
\hline & & Formicidae sp3 & 3 \\
\hline & & Formicidae sp4 & 2 \\
\hline & & Formicidae sp5 & 7,9 \\
\hline & & Proctotrupidae sp1 & 6 \\
\hline & Isoptera & Termitidae sp1 & 2 \\
\hline & Lepidoptera & Lepidoptera sp1 & 5 \\
\hline & & Lepidoptera sp2 & 6 \\
\hline & Psocoptera & Psylliosocidae sp1 & $1,5,6,7,8,9$ \\
\hline & & Psylliosocidae sp2 & 5 \\
\hline & Thysanoptera & Phloeotripidae sp1 & $3,4,6$ \\
\hline
\end{tabular}

Tabela 2. Valores de tamanho, Distância e índice de Diversidade de Shannon (H’) dos capões amostrados na região da Base de Estudos do Pantanal ( $19^{\circ} 34^{\prime} 34,84^{\prime \prime}$ S e $57^{\circ} 1^{\prime} 6,98^{\prime \prime}$ O), Corumbá, MS, em outubro de 2011.

\begin{tabular}{cccc}
\hline Capão & Área (ha) & Distância (km) & Shannon \\
\hline 1 & 0,48 & 1,61 & 1,0 \\
2 & 0,45 & 1,83 & 0,76 \\
3 & 0,40 & 1,76 & 0,987 \\
4 & 0,60 & 1,47 & 0,759 \\
5 & 1,38 & 0,72 & 1,27 \\
6 & 0,77 & 0,51 & 1,17 \\
7 & 0,52 & 0,72 & 1,032 \\
9 & 0,55 & 0,97 & 1,086 \\
\hline
\end{tabular}



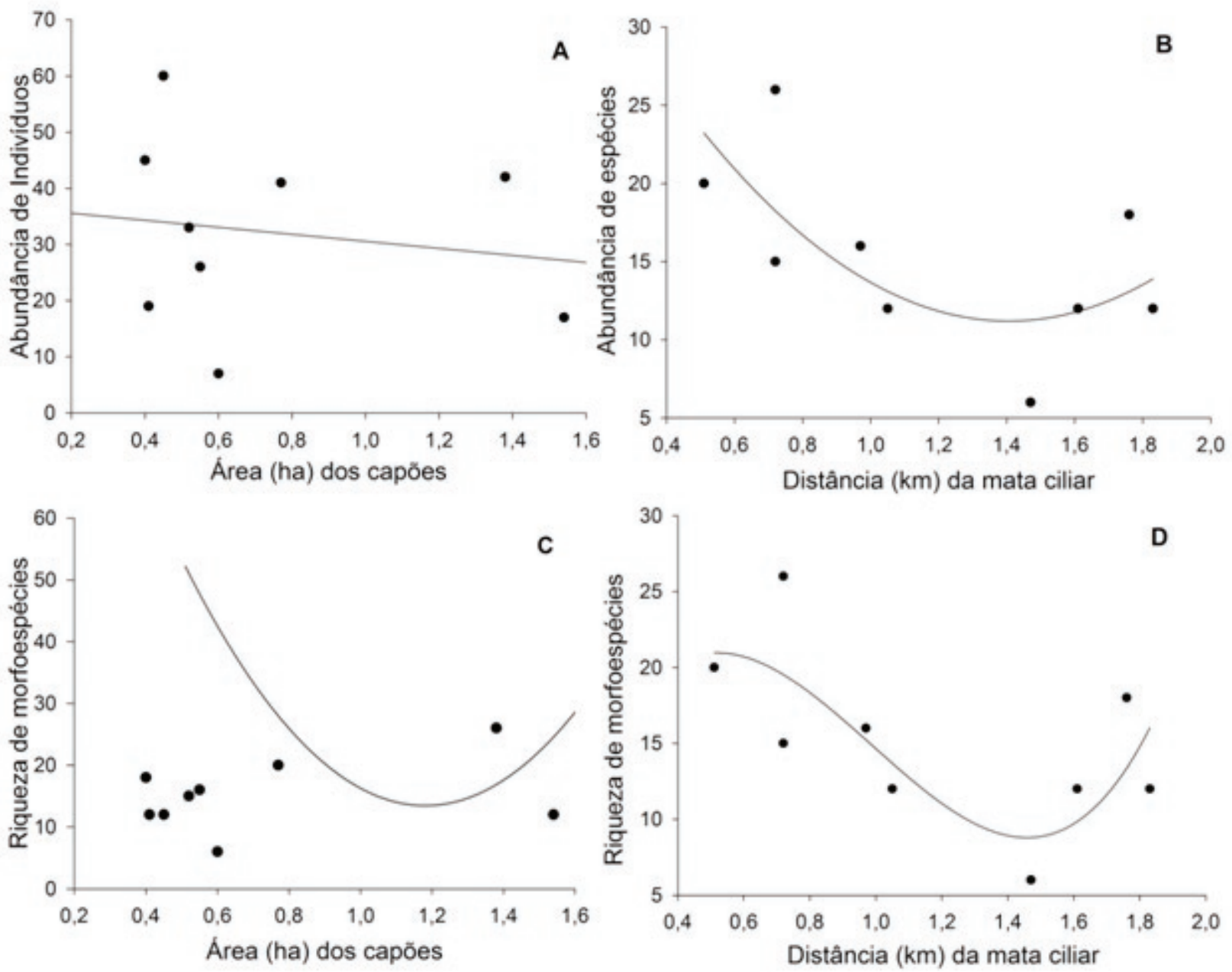

Figura 1. Relação entre Abundância de indivíduos e área $\left(A, R^{2}=0,02, F=0,18, p=0,02\right)$, Distância $\left(B, R^{2}=0,46, F=2,58\right.$, $\left.p=0,02\right)$, Riqueza de espécies e área $\left(C, R^{2}=0,65, F=3,21, p=0,05\right)$ e Distância $\left(D, R^{2}=0,55, F=2.09, p=0,21\right)$ de artrópodes de solo em capões próximos a Base de Estudos do Pantanal ( $19^{\circ} 34^{\prime} 34.84^{\prime \prime S}$ e $\left.57^{\circ} 1^{\prime} 6.98^{\prime \prime O}\right)$, Corumbá, MS, em outubro de 2011.

A

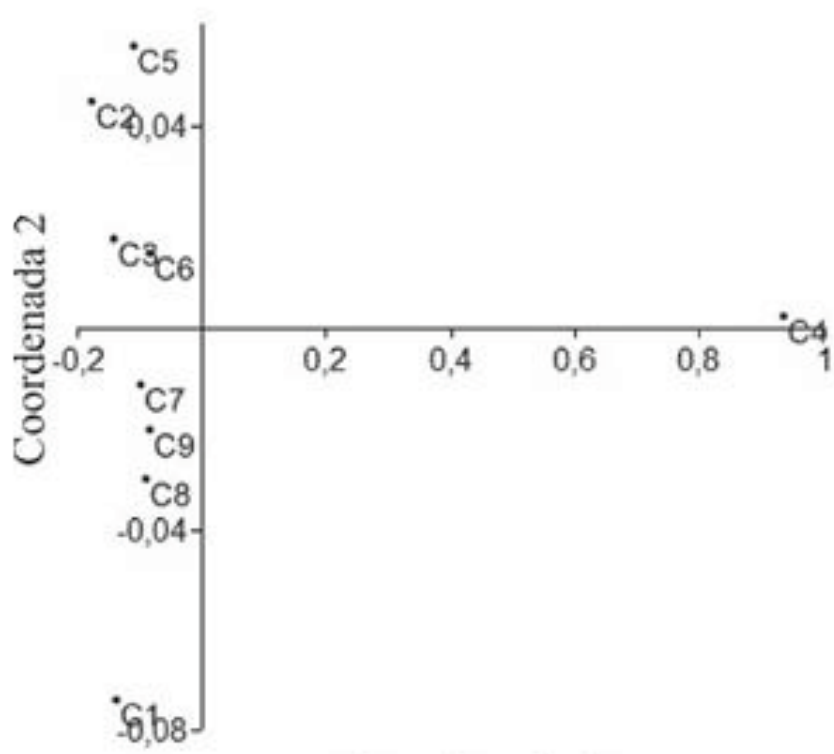

Coordenada 1
B

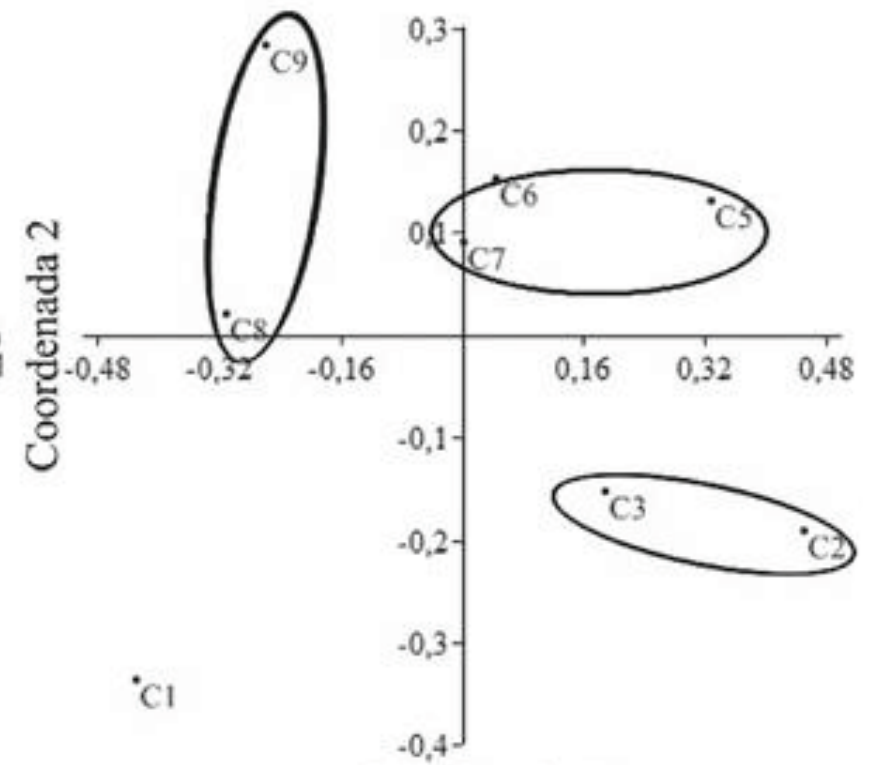

Coordenada 1

Figura 2. (A) Ordenação (NMDS) da comunidade de artrópodes de solo e serrapilheira em nove capões em relação ao tamanho (hectare) e distância da mata ciliar $(\mathrm{km})$ próximo a região da Base de Estudos do Pantanal $\left(19^{\circ} 34^{\prime} 34,84^{\prime \prime} \mathrm{S}\right.$ e $\left.57^{\circ} 1^{\prime} 6,98^{\prime \prime} \mathrm{O}\right)$, Corumbá, MS, em outubro de 2011. (B) Ordenação (NMDS) da comunidade de artrópodes de solo e serrapilheira em oito capões (capão 4 intensamente perturbado por pisoteio excluído)

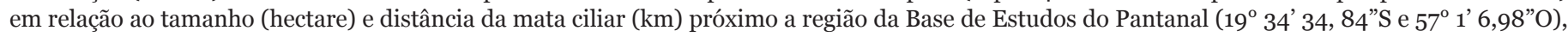
Corumbá, MS, em outubro de 2011. Elipses indicam capões mais próximos em distância ou em tamanho em relação à comunidade de artrópodes. 
biotaneotropica.org.br/v1on2/en/abstract?inventory+bnoo 210022010 [12/10/2011].

Bordignon, L., D. Moreira, T.F. Chupel \& C.M.S.C. Brazão, 2007. Ilhas vegetacionais no pantanal Matogrossense: um teste da Teoria de Biogeografia de Ilhas. Revista Brasileira de Biociências, 5: 387-389.

Borror, D.J. \& D.M. DeLong, 1988. Introdução ao estudo dos insetos. $1^{\circ}$ Edição. Edgard Blucher. São Paulo, SP. 653p.

Castilho, A.C.C., M.I. Marques, J. Adis \& A.D. Brescovit, 2005. Distribuição sazonal e vertical de Araneae em área com predomínio de Attalea phalerata Mart. (Arecaceae), no Pantanal de Poconé, Mato Grosso, Brasil. Amazoniana, 18: 215-239.

Correia, M.E.F. 2002. Relações entre a diversidade da fauna de solo e o processo de decomposição e seus reflexo sobre estabilidade dos ecossistemas. Seropédica: EMBRAPA (Centro Nacional de Pesquisa de Agrobiologia, EmbrapaCNPAB, Documentos, 156), 33p.

Corrêa, M.M., W.D. Fernandes \& I.R. Leal, 2006. Diversidade de formigas epigéicas (Hymenoptera, Formicidae) em Capões do Pantanal Sul Matogrossense: Relações entre riqueza de espécies e complexidade estrutural da área. Neotropical Entomology, 35: 724-730.

Debinsky, D.M. \& R.D. Holt, 2000. A Survey and overview of habitat fragmentation experiments. Conservation biology, 14:342-355.

Frizon, S., 2007. Diversidade de espécies arbóreas em Capões, Pantanal Sul: Relações com a área e o isolamento das manchas florestais. Dissertação (Mestrado em Ecologia e conservação) - Universidade Federal de Mato Grosso do Sul. 44p.

Gomes, A.A., R.M. Mussury, S.P.Q. Scalon, F. Watthier, K.A.A. Cunha \& H.S. Filho. 2007. Avaliação do impacto da fragmentação de florestais nativas sobre a mesofauna edáfica na região de Dourados-MS. Ciência Agrotécnica, 31: 612618.

Hughes, J.B., G.C. Daily \& P.R. Ehrlich, 2000. Conservation of insect diversity: a Habitat approach. Conservation Biology. 14: 1788-1797.

Hunter, M.D., 2002. Landscape structure, habitat fragmentation, and the ecology of insects. Agricultural and Forest Entomology, 4: 159-166.

Illig, J., R.A. Norton, S. Scheu, \& M. Maraum, 2010. Density and community structure of soil- and bark-dwelling microarthropods along an altitudinal gradient in a tropical montane rainforest. Experimental Application Acarology. 52: 49-62.
Junk, W.J., P.B. Bayley \& R.E. Sparks, 1989. The flood pulse concept in river-floodplain systems. Canadian Special Publication of Fisheries and Aquatic Sciences, 106: 110-127.

Krawchuk, M.A. \& P.D. Taylor, 2003. Changing importance of habitat structure across multiple spatial scales for three species of insects. Oikos, 103: 153-161.

Levins, R., 1969. Some demographic and genetic consequences of environmental heterogeneity for biological control. Bulletin of the Entomological Society America, 15: 237-240.

MacArthur, R.H. \& E.O. Wilson, 1967. The Theory of island biogeography. Princeton press, 224p.

Marques, M.I., J. Adis, G.B. dos Santos \& L.D. Batirrola, 2006. Terrestrial arthropods from tree canopies in the Pantanal of Mato Grosso, Brazil. Revista Brasileira de Entomologia 50: 257-267.

McIntyre, N.E., J. Rango, W.F. Fagan \& S.H. Faeth, 2001. Ground arthropod community structure in a heterogeneous urban environment. Landscape and Urban Planning, 52: 257-274.

Pott, A. \& V.J. Pott, 2009. Vegetação do Pantanal: fitogeografia e dinâmica. In Anais $2^{0}$ Simpósio de Geotecnologias no Pantanal. 1065-1076.

Ribas, C.R., T.G. Sobrinho, J.H. Schoereder, C.F. Sperber, C. Lopes-Andrade \& S.M. Soares, 2005. How large is large enough for insects? Forest fragmentation effects at three special scales. Acta Ecologica, 27:31-41.

Rubbo, M.J. \& J.M. Kiesecker, 2004. Leaf litter composition and community strucuture: translating regional species changes into local dynamics. Ecology, 85: 2519-2525.

Souza, R.C., M.E.F. Correia, M.G. Pereira, E.M.R. Silva, R.R. Paula \& L.F.T. Menezes, 2008. Estrutura da comunidade da fauna edáfica em fragmentos florestais na Restinga de Marambaia, RJ. Revista Brasileira de Ciências Agrárias, 3: 49-57.

Strong, Jr., D.R., \& J.R. Rey, 1982. Testing for MacArthur-Wilson equilibrium with the arthropods of the miniature Spartina archipelago at Oyster Bay, Florida. American Zoologist, 22: 355-360.

\section{Recebido em: 13/o3/2013}

Aceito em: 28/05/2013

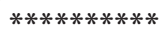

\section{Como citar este artigo:}

Aranda, R., 2013. Capões Como Ilhas para Artrópodes no Pantanal. EntomoBrasilis, 6(3): 173-177

Acessível em: http://www.periodico.ebras.bio.br/ojs/index.php/ebras/article/view/331. doi:10.12741/ebrasilis.v6i3.331
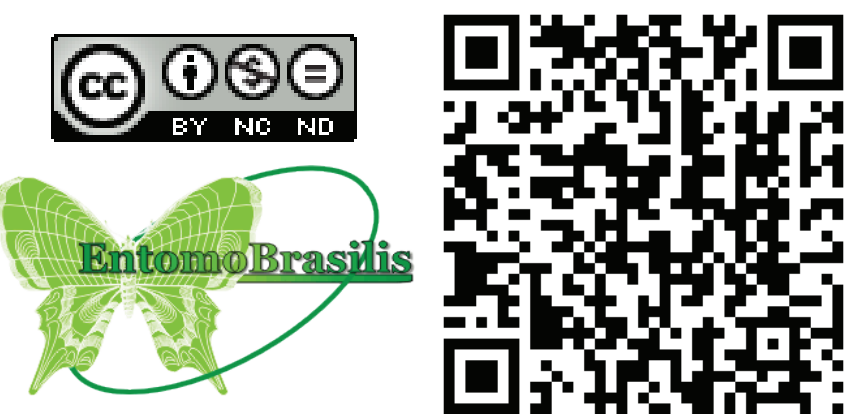